\title{
Single Data Copying for MPI Communication Optimization on Shared Memory System
}

\author{
Qiankun Miao $^{1}$, Guangzhong Sun ${ }^{1, \star}$, Jiulong Shan ${ }^{2}$, and Guoliang Chen ${ }^{1}$ \\ 1 Anhui Province-MOST Key Co-Lab of High Performance Computing \\ and Its Applications, Department of Computer Science, \\ University of Science and Technology of China, Hefei, 230027, P.R. China \\ ${ }^{2}$ Microprocessor Technology Lab, Intel China Research Center, Beijing, China \\ miao@mail.ustc.edu.cn, gzsun@ustc.edu.cn, jiulong.shan@intel.com, \\ glchen@ustc.edu.cn
}

\begin{abstract}
Shared memory system is an important platform for high performance computing. In traditional parallel programming, message passing interface (MPI) is widely used. But current implementation of MPI doesn't take full advantage of shared memory for communication. A double data copying method is used to copy data to and from system buffer for message passing. In this paper, we propose a novel method to design and implement the communication protocol for MPI on shared memory system. The double data copying method is replaced by a single data copying method, thus, message is transferred without the system buffer. We compare the new communication protocol with that in MPICH an implementation of MPI. Our performance measurements indicate that the new communication protocol outperforms MPICH with lower latency. For Point-to-Point communication, the new protocol performs up to about 15 times faster than MPICH, and it performs up to about 300 times faster than MPICH for collective communication.
\end{abstract}

\section{Introduction}

Message Passing Interface (MPI) [1] is a standard interface for high performance parallel computing. MPI can be used on both distributed memory multiprocessor and shared memory multiprocessor. It has been successfully used to develop many parallel applications on distributed memory system.

Shared memory system is an important architecture for high performance computing. With the advent of multi-core computing [4], shared memory system will draw more and more attention. There are several programming models for shared memory system, such as OpenMP [2] and Pthread [3. However, we still need to program on shared memory system with MPI. First, it is required to reuse the existing MPI code on distributed memory system for quickly developing applications on shared memory system. Second, applications developed using MPI are compatible across a wide range of architectures and need only a few modifications for performance tune-up. Third, the future computation will

\footnotetext{
* Corresponding author.
} 
shift to multi-core computation. Consequently, exploiting the parallelism among these cores becomes especially important. We must figure out how to program on them to get high performance. MPI can easily do such works due to its great success on parallel computing today.

It is difficult to develop an efficient MPI application on shared memory system, since the MPI programming model does few considerations of the underlying architecture. In the default communication protocol of MPI, receiver copies data from system buffer after sender copied the data to the system buffer. Usually a locking mechanism is used for synchronization on shared memory system, which has a high overhead. Therefore, MPI programs suffer severe performance degradation on shared memory system. In this paper, we propose techniques to eliminate these performance bottlenecks. We make use of the shared memory for direct communication bypass buffer copying instead of double data copying. Meanwhile, A simple busy-waiting polling method is used to reduce expense of locking for synchronization. To evaluate these techniques, some experiments are carried out on basic MPI primitives. We make comparisons of the performance between the new implementation and the original implementation in MPICH 5]. Results indicate that the new implementation is able to achieve much higher performance than MPICH do.

There are other works studying on the optimized implementation of the communication in MPI programs on shared memory system. A lock-free scheme is studied on NEC SX-4 machine [6]. They use a layered communication protocol for a portable consideration. A shared memory communication device in MPICH is conducted for Sun's UltraEnterprise 6000 servers [7]. They use a static memory management similar as we describe in Section 2. TMPI [8 use a threadlevel approach for communication optimization. TMPI can achieve significant performance advantages in a multiprogrammed environment. We consider the condition only a process per processor. This condition is usually happened in real computation. Our implementation is not restricted to a certain machine. We could achieve lower latency than the native MPI implementation.

The rest of the paper is organized as follows. Next section introduces the default communication implementation of MPI and discusses its drawbacks on shared memory system. Section 3 describes the design and implementation of the new approach used in this paper, which makes efficient communication on shared memory system. Section 4 describes our evaluation methodology and presents the evaluation results. Section 5 concludes the paper and presents the future work.

\section{Motivation}

In traditional implementation of MPI, a general shared memory device is needed, which can be used on many differently configured systems. There is a limitation that only a small memory space shared by all processes can be allocated on some systems. As a result, the communication between two processes is through a shared memory system buffer [9]. The system buffer can be accessed by all the processes in a parallel computer. We indicate this data transmission procedure in Fig. 1 


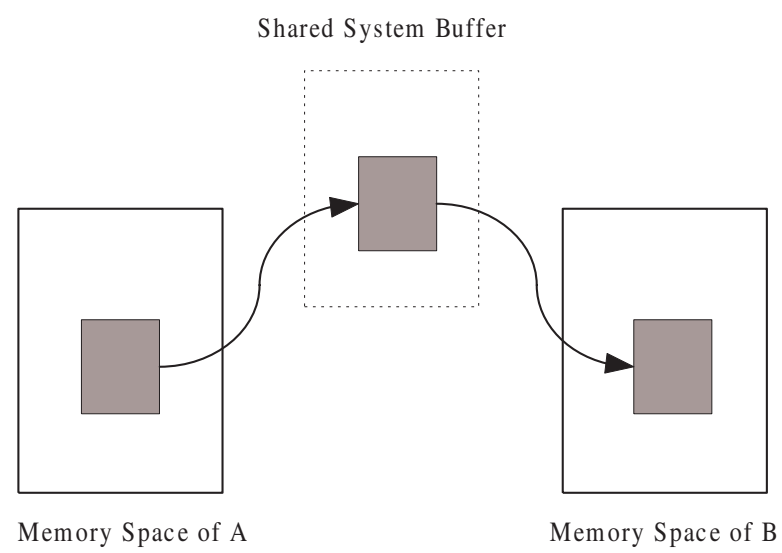

Fig. 1. Communication in original MPI, the message packet is transmitted by copying them into and out of the shared system buffer

A typical implementation of communication between two processes could be as follows. Suppose process A intends to send a message to process B. We use A to denote process $\mathrm{A}$ and $\mathrm{B}$ to denote process $\mathrm{B}$ following. There is a free shared memory segment pre-allocated and a synchronization flag can be accessed by A and B. The synchronization flag is used to indicate whether the shared memory segment is ready for A to write to or for B to read from. At the beginning, A checks the synchronization flag. If the shared memory segment is ready for writing, A copies data to be transmitted from its user buffer into the shared memory segment. Then A sets the synchronization flag after finishing the data copying. This indicates that the data in the shared memory segment is ready to be copied to B's user buffer. A can either wait for an acknowledgement after copying or it can continue execution. If the shared memory segment is not ready for A, it chooses a back-off strategy, and tries again later. Meanwhile, B can check for a forthcoming data packet by probing the synchronization flag. When it finds the flag is set, B copies the data in shared memory segment to its own user buffer. After the data transmitted completely, B clears the synchronization flag. At this time new communication can start. MPICH implements the above mechanism as an abstract device interface ch_shmem [7.

The communication strategy above is inefficient for a few reasons.

1. A double data copying protocol is required for each data exchange. But this is unnecessary and will increase the burden of memory/cache system. It will result in high latency of the communication.

2. A shared system buffer is required where the data is copied to and from. This is an extreme waste of capacity of memory. On shared memory system, local memory and cache capacity as well as aggregate bandwidth will limit the scalability of MPI implementation. 
3. The cost of back-off strategy for synchronization is extremely large. The synchronization cost can adversely affect communication performance for short message.

4. Furthermore, for those collective communications (e.g. MPI_Bcast), the extra copy and complex synchronization will aggravate these problems.

In a word, the critical problem for the communication on shared memory system is how to reduce the memory/cache capacity and the waiting time for synchronization. In the next section, we will propose techniques to solve these problems.

\section{Design and Implementation}

To solve the problems existing in MPI for shared memory system, we design new communication protocols. We use primitives (IPC/shm) to create process level shared memory segment for message to be transmitted. Communication among processes uses a single data copying protocol. Considering usually only one process per processor we choose a simple busy-waiting polling strategy for synchronization.

\subsection{Optimized Communication Protocol}

In Section 2, we have described the default implementation of communication in MPI. In order to transmit data among processes, a double data copying protocol is required. One is used to copy the data into shared memory, and the other is used to copy the data from the shared memory. However, the double data copying can be reduced to only one data copying when the sender process need retain an independent copy of the data. Even no data copying is required when the sender process needn't retain a copy of the data or all the processes share the same data structure and do operation on disjoint parts of the data structure. We can allocate a shared memory segment for the data to be transmitted. Thus, every process can access this shared segment. Data in the shared memory segment can be copied to the receiver process's user buffer either in the shared memory or in its private space. If all the processes deal with disjoint parts of the same data structure, we need no data transmission because after one process updated the data all other processes can see the update immediately and they can use $\mathrm{IPC} /$ shm to directly read the data. This technique is able to obtain lower latency and less memory consumption, because it needs only one or no data copying and no extra system buffer. Fig. 2 illustrates this technique.

According to the above discussion, we can model the required time for sending a message packet with size of $\mathrm{n}$ bytes by the following formulas. We use $T_{\text {original }}$ to indicate the communication time of the original version and $T_{\text {optimized }}$ to indicate that of our optimized implementation.

Original MPI device using two data copying:

$$
T_{\text {original }}=2 T_{\text {datacpy }}+T_{\text {syn }}
$$




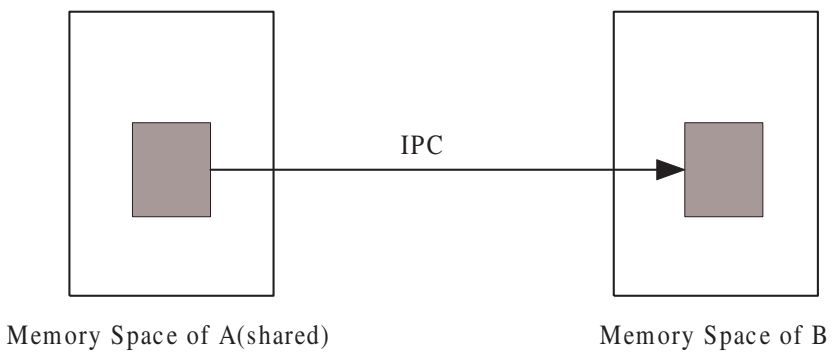

Fig. 2. Optimized communication with only a single buffer copying by employing IPC

Optimized implementation using single data copying:

$$
T_{\text {optimiezed }}=T_{\text {datacpy }}+T_{\text {alloc }}+T_{\text {free }}+T_{\text {bwsyn }}
$$

Optimized implementation using no data copying:

$$
T_{\text {optimiezed }}=T_{\text {alloc }}+T_{\text {free }}+T_{\text {bwsyn }}
$$

Where $T_{\text {datacpy }}$ represents the time for one data copying, $T_{\text {syn }}$ represents the time for communication synchronization in original version, $T_{\text {alloc }}$ and $T_{\text {free }}$ represent the time for shared memory allocating and deleting respectively, $T_{\text {bwsyn }}$ represents the time for communication synchronization using busy-waiting strategy in our implementation.

In general case, the cost of the shared memory segment allocation and free is very little compared with the cost of one data copying. So, $T_{\text {original }}$ is larger than $T_{\text {optimized }}$. This indicates that we could achieve higher performance using the optimized communication protocol.

\subsection{Busy-Waiting Polling Strategy}

A synchronization mechanism is used to prevent the processes from interfering with each other during the communication procedure. It defines the time when sender process makes data ready and the time when receiver process completes the data copying. Usually the mechanism is provided by locks and semaphores on shared memory system. Unfortunately, typical implementations of lock tend to cause a system call, which is often very time-consuming. So, the cost of synchronization by using a lock is too expensive to provide high performance. When the lock is not free, a back-off strategy is used. This may delay the starting time of communication. Though the lock is already free, it may still need to wait for the process to be active again.

With the assumption that there is usually only one application process per processor and processor resources are not needed for other tasks, we use a simple busy-waiting polling approach to replace exponential back-off strategy. An application process repeatedly tests the synchronization flag as frequently as possible 
to determine when it may access the shared memory. Once the process has found the synchronization flag switched, it would immediately detect the change. Consequently, the data transmission can start without delay. This polling strategy would reduce the time for synchronization on shared memory system when there is only one process per processor.

\section{Performance Evaluation}

We conduct experiments to study the performance of our implementation. Latency time is collected for multiple repetitions of each operation over various message sizes between 0 byte and 1 megabytes. We use latency time here to denote the total transmission time of a message among processes. For 0 byte message, latency time is only the overhead of communication start-up and end. All the results given are averaged over multiple runs.

\subsection{Platform Configuration}

The target system is a 16-way shared memory multiprocessor system running Suse Linux 9.0 platform. It has 16 x 86 processors running at $3.0 \mathrm{GHz}, 4$ levels of cache with each $32 \mathrm{MB}$ L4 cache shared amongst 4 CPUs. As for the interconnection, the system uses two $4 \mathrm{x} 4$ crossbars. We use MPICH-1.2.5.2 (affiliated with device ch_shmem and gcc 3.3.3) library form Argonne National Laboratory to generate the executables. Each process is bound to a processor to prevent operating system from migrating processes among processors.

\subsection{Performance Evaluation Results}

Point-to-Point communication transmits data between a pair of processes. We choose the standard MPI_Send/Recv for our test. Collective communication is often used in real applications. Usually it involves a large number of processes. This means that there is a large amount of data to be transmitted among the involved processes. So, its performance heavily depends on the implementation. We investigate MPI_Bcast, MPI_Gather, MPI_Scatter, MPI_Alltoall, MPI_Reduce to evaluate the collective communication with new communication approach. We only present the results of MPI_Bcast due to the limited paper size. In MPI_Bcast a root process broadcasts a message to all the other processors.

We present the experimental results in Fig. 3. We compare two implementations of Send/Recv in the left of Fig. 3. one is the default version in MPICH which is labeled Original Send/Recv, the other is the optimized version which is labeled New Send/Recv. In the right of Fig. 3, we compare two implementations of Bcast which are labeled Original Bcast and New Bcast respectively.

From Fig. 3. we can see that our new implementation outperforms the default implementation in MPICH. Our implementation has lower latency on various size message for both point-to-point communication and collective communication. Our implementation is almost six times faster than MPICH for short 

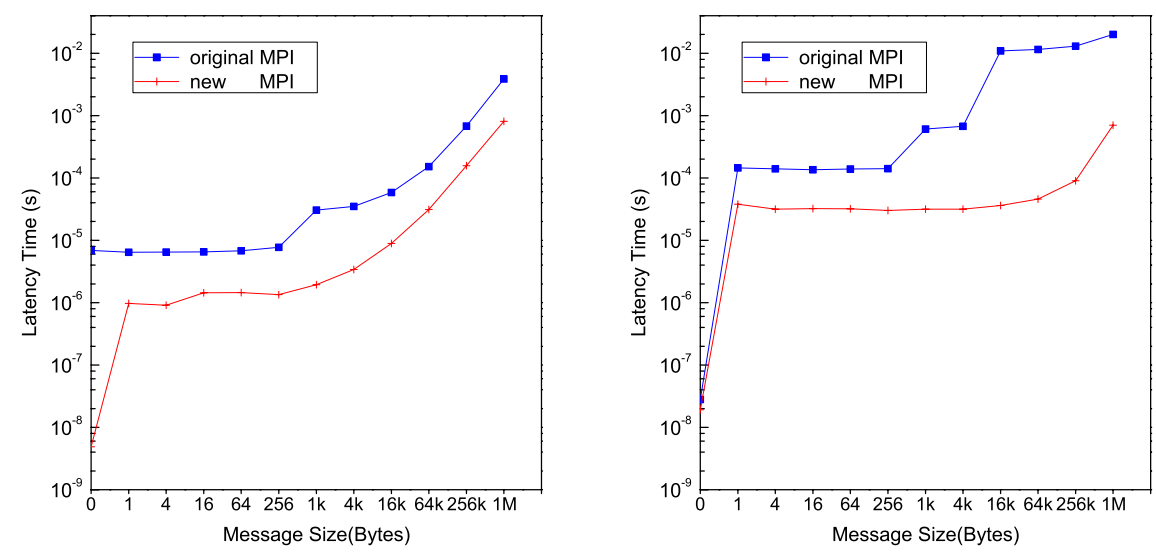

Fig. 3. Left: Latency time of original MPICH Send/Receive (upper line) and optimized implementation (lower line). Right: Latency time of original MPICH Bcast (upper line) and optimized implementation (lower line).

message and up to fifteen times faster for long message. Our implementation gains great success for collective communication. The optimized implementation is about five times faster than MPICH for short message. It is two orders of magnitude faster than MPICH when the message size is larger than $1 \mathrm{kBytes}$. For message size from 1 bytes to $4 \mathrm{kBytes}$, the transmission time of broadcast is almost no increase in the new implementation. That's because all other processes can directly copy data from the sender process's user buffer simultaneously, and the transmission time is determined by the cost of memcpy() which is almost same for various short data sizes. As the message size is large, memory bandwidth contention will limit the communication performance, the transmission time increases with message size. When the message is so large that the system buffer pre-allocated for communication of the original MPI implementation can't hold the total message at one time, it is required to split the message to several small pieces and transmits one piece at a time. We can see a step-up increase of the transmission time in MPICH from Fig. 3. However, all receivers can still directly copy from the sender's buffer in the optimized implementation, since the optimized implementation isn't limited on message size.

\section{Conclusion}

In this paper, we present an optimized implementation of the communication significantly improves the performance of MPI communication on shared memory system. The optimized methods include a single data copying protocol and a busy-waiting polling strategy for synchronization. Some experiments are conducted to evaluate the performance of a few basic MPI primitives with the optimized communication. The experimental results indicate that the primitives 
with optimized communication achieved lower latency than the native version in MPICH.

For future work, we intend to use the methods to develop more real-world applications on shared memory system and make a source to source translator to translate the MPI programs written for distributed system into an efficient shared memory version. We will also compare the overall performance of an application written using optimized message passing with that of an application written using shared memory programming model such as OpenMP.

\section{Acknowledgements}

This work is supported by the National Natural Science Foundation of China No.60533020. This work is partially finished at Intel China Research Center. We would like to thank the anonymous referees for their useful suggestions to improve the presentation of this paper.

\section{References}

1. The MPI Forum. The MPI Message-Passing Interface Standard. http://www.mpiforum.org/. (1995).

2. OpenMP Standards Board. OpenMP: A Proposed Industry Standard API for Shared Memory Programming. http://www.openmp.org/openmp/mpdocuments/paper/paper.html. (1997).

3. Pthread interface. ANSI/IEEE Standard 1003.1. (1996)

4. M. Greeger. Multicore CPUs for the Masses. ACM Queue, 3(7) (2005):63-64

5. W. Gropp, E. Lusk: Skjellum A, Doss N. MPICH: A high-performance, portable implementation for the MPI message-passing interface. Parallel Computing 1996; 22, (1996) pp. 789-928.

6. W. Gropp, E. Lusk: A high-performance MPI implementation on a shared-memory vector supercomputer. Parallel Computing, 22, (1997) pp. 1513-1526.

7. B. V. Protopopov, A. Skjellum: Shared-memory communication approaches for an MPI message-passing library. Concurrency: Practice and Experience, 12(9), (2000) pp. $799-820$.

8. H. Tang, K. Shen, and T. Yang: Compile/Run-time Support for Threaded MPI Execution on Multiprogrammed Shared Memory Machines. In Proceedings of ACM SIGPLAN Symposium on Principles and Practice of Parallel Programming, (1999) pp. 107-118.

9. D. Buntinas, M. Guillaume, W. Gropp: Data Transfers between Processes in an SMP System: Performance Study and Application to MPI. International Conference on Parallel Processing (ICPP), Columbus, Ohio, USA, (2006), pp. 487-496. 